\section{Erste Studien im Internet: Mit Antikörpern gegen Allergien}

\author{
Auf einer neu eingerichteten Homepage können sich Ärzte \\ über einen neuartigen Ansatz in der Asthma- und Allergietherapie \\ informieren. Vorgestellt wird der erste monoklonale Antikörper \\ gegen Immunglobulin E mit dem Arbeitsnamen rhu-Mab/E25. \\ Der Fortgang der Phase-III-Studien an Asthmapatienten \\ kann jetzt im Internet auf dem jeweils aktuellen Stand \\ nachvollzogen werden.
}

als auch Spätphase hat. Der Abfall der FEV1-Werte war in der Früh- ebenso wie in der Spätphase unter rhuMab/E25 nach Provokationstests signifikant schwächer ausgeprägt als unter Plazebo. Des weiteren fiel die Bronchokonstriktion in der Spätphase der allergischen Reaktion um 60\% geringer aus. Bei einem PC20-Provokationstest konnte die Methacholindosis 24 Stunden nach Allergenkontakt unter rhu-Mab/E25 um das 1,6fache erhöht werden.

Darüber hinaus hat der Einsatz von rhu-Mab/E25 einen positiven Einfluß auf den Entzündungsprozeß bei Patienten mit chronisch persistierendem Asthma. Dies belegen auch SputumUntersuchungen, bei denen sich 24 Stunden nach Allergenkontakt die Zahl der Eosinophilen um das elffache verringerte

Auch andere Allergie-

Zur Zeit laufen bei der Firma Novartis Pharma internationale multizentrische Phase-III-Studien mit dem Anti-IgE-Antikörper in den Indikationsgebieten allergisches Asthma und saisonal bedingte allergische Rhinitis. Der Wirkstoff mit dem vorläufigen Namen rhuMab/E25 hat zwei Vorteile:

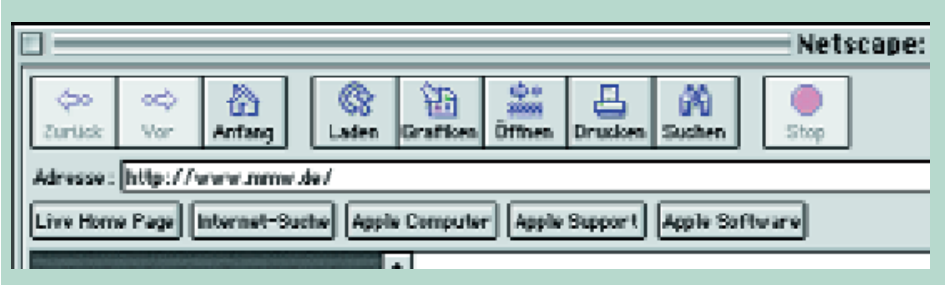

Allergie im Internet
Er wirkt gegen alle IgEvermittelten Allergien, unabhängig von der Art des Allergens, und beeinflußt sowohl die Früh- als auch die Spätphase der allergischen Reaktion.

\section{Ziel der Antikörpertherapie:}

Allergiekaskade dauerhaft stoppen

Die bereits vorliegenden Ergebnisse der Phase-I- und -II-Studien lassen schon jetzt auf eine Bereicherung der Allergie- bzw. Asthmatherapie hoffen: $\operatorname{IgE}$ spielt bekanntlich eine wichtige Rolle in der Pathogenese allergischer Erkrankungen: Das Immunglobulin führt durch Bindung an die Mastzellen zur Freisetzung von Histamin und anderen Mediatoren wie Leukotrienen, Interleukinen oder Zytokinen. Der Wirkstoff rhu-Mab/E25 bindet dabei Allergen-unspezifisch die freien IgEAntikörper im Serum.

Ziel der E25-Therapie ist es, die Allergiekaskade dauerhaft zu unterbrechen und so die Bedarfsmedikation und den Verbrauch an Kortikosteroiden auf ein Minimum zu reduzieren. Gleichzeitig besteht damit erstmals die Möglichkeit, das inflammatorische Remodelling und damit langfristig den chronischen Verlauf des Asthmas zu beeinflussen.

In einer plazebokontrollierten Studie, an der 316 Asthmapatienten teilnahmen, konnte unter Gabe von rhuMab/E25 der Verbrauch an oralen sowie inhalativen Kortikosteroiden signifikant gesenkt werden. Darüber hinaus kam es zu einer ebenfalls signifikanten Reduktion des Bedarfs an Notfallmedikamenten (z.B. kurzwirksame Beta2Mimetika).

\section{Einfluß auf die Früh- und Spätreaktion}

In einer weiteren plazebokontrollierten Studie an Asthmapatienten zeigte sich, daß rhu-Mab/E25 einen positiven Einfluß sowohl auf die allergische Früh- formen scheinen auf den neuen Wirkstoff anzusprechen. In einer offenen Verträglichkeitsstudie an 47 Patienten mit perennialer allergischer Rhinitis wurde die Hautreaktion bei Allergenkontakt vor und nach der Behandlung mit rhu$\mathrm{Mab} / \mathrm{E} 25$ untersucht. In der Gruppe, die das Medikament niedrig dosiert erhielt, reduzierte sich die Quaddelgröße um $60 \%$, in der Hochdosis-Gruppe wurde ein Rückgang sogar um $80 \%$ erzielt.

\section{Hintergrundinformationen zum Herunterladen}

Ausführliche Informationen zu Wirksamkeit, Indikation und Verträglichkeit sowie eine detaillierte Auflistung der Studienergebnisse der Phase I und II sind auf der E25-Homepage abrufbar. Die Originalstudien können bei Interesse als zip-Datei auf den heimischen Rechner geladen werden.

rhu-Mab/E25 wird biotechnisch hergestellt, auch dazu finden sich Erläuterungen im Internet. Sobald neue Studienergebnisse vorliegen, werden die Internetseiten aktualisiert, so daß der Fortgang der Studien direkt online verfolgt werden kann. Die Adresse der Homepage: www.e25.de

(prk/bk) 